\title{
Glucose-6-Phosphate dehydrogenase deficiency genetic variation in vivax malaria patients from Thai-Myanmar and Thai-Malaysian border
}

\author{
Nutnicha Suphakhonchuwong, Nammon Rattaseree, Pattranit Rodjanadarawong, Jiraporn Kuesap \\ Pharmacology and Toxicology, Graduated Programs in Biomedical Sciences, Faculty of Allied Health Sciences, \\ Thammasat University, Phahonyothin Road, Klonglung, Pathum Thani Thailand 12120, Thailand
}

Plasmodium vivax is the most widespread parasite causing human malaria and the major cause of malaria in most of Asia and Latin America. Anemia is considered as major important markers of severity during the clinical course of severe Plasmodium vivax malaria. The hypnozoitocidal and schizonticidal anti-malarial drug for the radical cure of vivax malaria infection is primaquine. The main adverse effect of primaquine is hemolytic anemia in individual glucose-6-phosphate dehydrogenase (G6PD) deficiency. The objective of this study was to determine the prevalence of G6PD variants in malaria patients residing along Thai-Mynmar and Thai-Malaysian border. Total of one hundred and seventy-one blood samples with Plasmodium vivax identification were collected from Kanchanaburi and Ranong province (Thai-Mynmar), and Yala province (Thai-Malaysian). The G6PD deficiency variants were analyzed by polymerase chain reaction and restriction fragment length polymorphism (PCR/RFLP). Three specific primers of each G6PD deficiency variants mostly found in Thailand were investigated including Mahidol variants (487G $>A$ ), Chinese-4 variants $(392 \mathrm{G}>\mathrm{T})$, and Viangchan variants $(871 \mathrm{G}>\mathrm{A})$. Approximately five percent of isolates were identified as G6PD deficiency with Mahidol and Chinese 4 variant. According to Thai-myanmar border, 5.4\% of Mahidol variant and $0.7 \%$ of Chinese 4 were found. Whereas, Thai-Malaysian border only one variant with $2.3 \%$ of were found. The coexistence might be related to high hemolytic risk in malaria patients leading to severity due to insufficient of G6PD enzyme in patients who live in different endemic area. No Viangchan variants was found. Dosage regimen of primaquine for treatment of Plasmodium vivax malaria in this population may need to be optimized, based on G6PD variants information. With this limited G6PD variants, other variation of G6PD should be required to obtain accurate genetic mapping of G6PD variants in malaria patients residing in malaria endemic areas along Thai-neighboring borders. 